\title{
Laplace Substitution - Variational Iteration Method for Solving Goursat Problems Involving Mixed Partial Derivatives
}

\author{
Ali Al-Fayadh, Dina Saad Faraj* \\ Email address: \\ aalfayadh ayahoo.com (A. Al-Fayadh), dina_saad2015@yahoo.com (D. S. Faraj) \\ ${ }^{*}$ Corresponding author
}

Department of Mathematics and Computer Applications, College of Science, Al-Nahrain University, Baghdad, Iraq

\section{To cite this article:}

Ali Al-Fayadh, Dina Saad Faraj. Laplace Substitution - Variational Iteration Method for Solving Goursat Problems Involving Mixed Partial Derivatives. American Journal of Mathematical and Computer Modelling. Vol. 4, No. 1, 2019, pp. 16-20.

doi: 10.11648/j.ajmcm.20190401.12

Received: February 26, 2019; Accepted: April 4, 2019; Published: May 10, 2019

\begin{abstract}
This paper will investigate a method to achieve the exact solution of special type of nonlinear partial differential equations (NLPDEs) involving mixed partial derivatives. This proposed method named as Laplace substitution - Variation iteration method (LS-VIM). The method exploits the properties of Laplace substitution method and the Variational iteration method to find the exact solution for Goursat problem involving mixed partial derivatives. In addition, this paper emphasizes the effectiveness of the LS-VIM by solving two examples. The results show that the exact solution can be achieved from a single iteration of the propose method.
\end{abstract}

Keywords: Laplace Transforms, Laplace Substitution Method, Variation Iteration Method, Mixed Partial Derivatives, Goursat Problems

\section{Introduction}

Nonlinear phenomena have important effects on various fields of applied mathematics and science. These phenomena frequently modeled through NLPDEs. NLPDEs involving mixed partial derivatives occur naturally in different fields of science, physics and engineering. The comprehensive applicability of these equations has gained so much attention from many mathematicians and scientists. However, it is still a huge problem to get the exact or approximate solutions for most models of these equations.

The Goursat partial differential equation arises in linear and nonlinear PDFs with mixed derivatives in the study of wave phenomena and it considered as a second order hyperbolic partial differential equation $[1,3,5]$. The standard form of the Goursat problems is given by:

$$
\begin{gathered}
u_{x t}=f\left(x, t, u, u_{x}, u_{t}\right), 0 \leq x \leq a \quad, 0 \leq t \leq b \\
u(x, 0)=g(x), u(0, t)=h(x) \\
g(0)=h(0)=u(0,0)
\end{gathered}
$$

Many numerical methods were established for solving the nonlinear type of Goursat problem such as Adomian decomposition method(ADM) [1], Homotopy analysis method(HAM) [9], Variation iteration method (VIM) [10], Two-dimensional differential transform method [8], Modified Variational iteration methods [6], Reduced differential transform method (RDTM) [7] and Finite Difference Method (FDM) [14]. All these methods have reached the exact solution for solving Goursat problems.

As mentioned above, VIM was used to solve PDE numerically. Benefitting the exciting features of this method, it combined with Laplace transformation method to solve NLPDE's with a single variable of interest. This combination called The Laplace transform - Variation iteration method which was applied successfully and efficiently to solve NLPDEs [2]. However, this method is restricted in solving NLPDEs with one variable only.

In this paper, a new method is developed to solve special type of NLPDEs with two variables. This method is consists of combining the Laplace Substitution Method (LSM) with the VIM to solve NLPDEs that include two variables. The 
Laplace Substitution Method (LSM) [4, 11-14] has been used to solve linear and nonlinear PDEs involving combined partial derivatives. The proposed combined method named as LS-VIM. This paper aims to investigate the applicability and the effectiveness of the suggested method to find the exact solution of Goursat NLPDE. The LS-VIM will be proposed in section 2. Furthermore, in section 3 LS-VIM will apply in two NLPDEs examples involving mixed partial derivatives. Some conclusions will be given in the last section.

\section{Laplace Substitution - Variational Iteration Method}

Laplace substitution is a mathematical technique used to solve a nonlinear equation with two independent variables. However, the solution reach a complex term that is difficult to solve. Therefore, the Laplace substitution method is combined with the VIM to simplified the solution and reach the exact solution. The aim of this section is to introduce the steps of achieving the LS-VIM for nonlinear partial differential equations involving mixed partial derivatives. The general form for the nonlinear, inhomogeneous partial differential equation rolling mixed partial derivatives and the initial conditions are shown as below:

$$
\begin{gathered}
\mathrm{Lu}(\mathrm{x}, \mathrm{t})+\mathrm{Ru}(\mathrm{x}, \mathrm{t})+\mathrm{Nu}(\mathrm{x}, \mathrm{t})=\mathrm{h}(\mathrm{x}, \mathrm{t}) \\
\mathrm{u}(\mathrm{x}, 0)=\mathrm{f}(\mathrm{x}), \quad \mathrm{u}_{\mathrm{t}}(0, \mathrm{t})=\mathrm{g}(\mathrm{t})
\end{gathered}
$$

Where $L=\frac{\partial^{2}}{\partial x \partial t}, \operatorname{Ru}(x, y)$ is the remaining linear operator, $\mathrm{Nu}$ represents a general nonlinear differential operator and $\mathrm{h}(\mathrm{x}, \mathrm{y})$ is the source term.

Equation (4) can written in the following form:

$$
\begin{aligned}
& \frac{\partial^{2} u}{\partial x \partial t}+\operatorname{Ru}(x, t)+N u(x, t)=h(x, t) \\
& \frac{\partial}{\partial x}\left(\frac{\partial u}{\partial t}\right)+R u(x, t)+N u(x, t)=h(x, t)
\end{aligned}
$$

Substituting $\frac{\partial \mathrm{u}}{\partial \mathrm{t}}=\mathrm{U}$ in equation (7), we get:

$$
\frac{\partial U}{\partial x}+R u(x, t)+N u(x, t)=h(x, t)
$$

Taking Laplace transform for equation (8) in respect with $\mathrm{x}$, we get:

$$
\begin{gathered}
\mathrm{L}\left\{\frac{\partial \mathrm{U}}{\partial \mathrm{x}}\right\}+\mathrm{L}\{\mathrm{Ru}(\mathrm{x}, \mathrm{t})\}+\mathrm{L}\{\mathrm{Nu}(\mathrm{x}, \mathrm{t})\}=\mathrm{L}\{\mathrm{h}(\mathrm{x}, \mathrm{t})\} \\
\mathrm{sU}(\mathrm{s}, \mathrm{t})-\mathrm{U}(0, \mathrm{t})=\mathrm{L}_{\mathrm{x}}[\mathrm{h}(\mathrm{x}, \mathrm{t})-\mathrm{Ru}(\mathrm{x}, \mathrm{t})-\mathrm{Nu}(\mathrm{x}, \mathrm{t})] \\
\mathrm{U}(\mathrm{s}, \mathrm{t})=\frac{1}{\mathrm{~s}} \mathrm{~g}(\mathrm{t})+\frac{1}{\mathrm{~s}} \mathrm{~L}_{\mathrm{x}}[\mathrm{h}(\mathrm{x}, \mathrm{t})-\mathrm{Ru}(\mathrm{x}, \mathrm{t})-\mathrm{Nu}(\mathrm{x}, \mathrm{t})]
\end{gathered}
$$

By taking the inverse Laplace transform for equation (11) with respect to $\mathrm{x}$ we get:

$$
\mathrm{U}(\mathrm{x}, \mathrm{t})=\mathrm{g}(\mathrm{t})+\mathrm{L}_{\mathrm{x}}^{-1}\left(\frac{1}{\mathrm{~s}} \mathrm{~L}_{\mathrm{x}}[\mathrm{h}(\mathrm{x}, \mathrm{t})-\mathrm{Ru}(\mathrm{x}, \mathrm{t})-\mathrm{Nu}(\mathrm{x}, \mathrm{t})]\right)
$$

Resubstitute the value of $U(x, t)$ in equation (12), we get:

$$
\frac{\partial \mathrm{U}(\mathrm{x}, \mathrm{t})}{\partial \mathrm{t}}=\mathrm{g}(\mathrm{t})+\mathrm{L}_{\mathrm{x}}^{-1}\left(\frac{1}{\mathrm{~s}} \mathrm{~L}_{\mathrm{x}}[\mathrm{h}(\mathrm{x}, \mathrm{t})-\mathrm{Ru}(\mathrm{x}, \mathrm{t})-\mathrm{Nu}(\mathrm{x}, \mathrm{t})]\right)
$$

Taking the Laplace transform of equation (13) with respect to $t$ we get:

$$
\begin{aligned}
& \mathrm{su}(\mathrm{x}, \mathrm{s})=\mathrm{f}(\mathrm{x})+\mathrm{L}_{\mathrm{t}}\left\{\mathrm{g}(\mathrm{t})+\mathrm{L}_{\mathrm{x}}^{-1}\left(\frac{1}{\mathrm{~s}} \mathrm{~L}_{\mathrm{x}}[\mathrm{h}(\mathrm{x}, \mathrm{t})-\mathrm{Ru}(\mathrm{x}, \mathrm{t})-\mathrm{Nu}(\mathrm{x}, \mathrm{t})]\right)\right\} \\
& \mathrm{u}(\mathrm{x}, \mathrm{s})=\frac{1}{\mathrm{~s}} \mathrm{f}(\mathrm{x})+\frac{1}{\mathrm{~s}} \mathrm{~L}_{\mathrm{t}}\left\{\mathrm{g}(\mathrm{t})+\mathrm{L}_{\mathrm{x}}^{-1}\left(\frac{1}{\mathrm{~s}} \mathrm{~L}_{\mathrm{x}}[\mathrm{h}(\mathrm{x}, \mathrm{t})-\mathrm{Ru}(\mathrm{x}, \mathrm{t})-\mathrm{Nu}(\mathrm{x}, \mathrm{t})]\right)\right\}
\end{aligned}
$$

Taking the inverse Laplace transform of equation (15) with respect to $t$, we get:

$$
u(x, t)=f(x)+L_{t}^{-1}\left\{\frac{1}{s} L_{t} g(t)+L_{x}^{-1}\left(\frac{1}{s} L_{x}[h(x, t)-R u(x, t)-N u(x, t)]\right)\right\}
$$

Derivative by $\frac{\partial^{2} \mathrm{u}}{\partial \mathrm{x} \partial \mathrm{t}}$ both sides of (16), we have:

$$
u_{x t}(x, t)-\frac{\partial^{2} u}{\partial x \partial t} f(x)-\frac{\partial^{2} u}{\partial x \partial t}\left[L_{t}^{-1}\left\{\frac{1}{s} L_{t} g(t)+L_{x}^{-1}\left(\frac{1}{s} L_{x}[h(x, t)-R u(x, t)-N u(x, t)]\right)\right\}\right]
$$

Using Langrange multiplier, the stationary conditions $1+\lambda=0$ follow immediately. This gives $\lambda=-1$.

Then the correction function of the iteration method:

$$
\left.u_{n}(x, t)-\int_{0}^{t} \int_{0}^{x}\left(\left(u_{n}\right)_{x \varepsilon}(x, \varepsilon)-\frac{\partial^{2} u}{\partial x \partial t} f(x)-\frac{\partial^{2} u}{\partial x \partial t}\left[L_{t}^{-1}\left(\frac{1}{s} L_{t}\left(g(t)-L_{x}^{-1}\left(\frac{1}{s} L_{x}[h(x, t)-R u(x, t)-N u(x, t)]\right)\right)\right)\right]\right) d \xi\right)
$$

The solution of Goursat problem presented in (6) is obtained (17) with the correction function (18). Finally, the solution $\mathrm{u}(\mathrm{x}, \mathrm{t})$ is given by:

$$
u(x, t)=\lim _{n \rightarrow \infty} u_{n}(x, t)
$$




\section{Applications}

Two examples are selected to evaluate the proposed method. These examples are used by previous authors to evaluate and explain their solutions [1, 6-10, 14], for that reason the same trend was followed in this paper. At the end of each example, the resulted solution is compared to the results obtained from previous references. The Langrange multiplier used in the solved example was $\lambda=-1$. In this section, we will apply the proposed LS-VIM to find the exact solution of the nonlinear Goursat problem.

\subsection{Example 1}

In this example, a nonlinear inhomogeneous Goursat problem used to check the efficiency of the LS-VIM. The used equation in this example is consist of two variables $\mathrm{x}$ and $t$ as shown in equation (20).

$$
\begin{aligned}
& u_{x t}=-u^{3}+x^{3}+3 x^{2} t+3 x t^{2}+t^{3} \\
& u(x, 0)=x, u(0, t)=t, u(0,0)=0
\end{aligned}
$$

Solution:

Applying Laplace transform on equation (28) with respect to t: (28)

$$
\begin{gathered}
S U(x, s)-U(x, 0)=L_{t}\left[t+\frac{x^{4}}{4}+x^{3} t+\frac{3}{2} x^{2} t^{2}+t^{2}\right]-L_{t}\left(L_{x}^{-1} \frac{1}{s} L_{x}\left(u^{3}\right)\right) \\
S U(x, s)=x+\left[\frac{1}{s^{2}}+\frac{x^{4}}{4}+\frac{x^{3}}{s^{2}}+\frac{3}{s^{3}} x^{2}+\frac{6}{s^{4}}\right]-L_{t}\left[L_{x}^{-2} \frac{1}{s} L_{x}\left(U^{3}\right)\right] \\
U(x, s)=\frac{x}{s}+\left[\frac{1}{s^{3}}+\frac{x^{4}}{4}+\frac{x^{3}}{s^{3}}+\frac{3 x^{2}}{s^{4}}+\frac{6}{s^{5}}\right]-\frac{1}{s} L_{t}\left(L_{x}^{-1} \frac{1}{s} L_{x}\left(u^{3}\right)\right)
\end{gathered}
$$

Using inverse Laplace transform on equation (31):

$$
\mathrm{U}(\mathrm{x}, \mathrm{t})=\left[\mathrm{X}+\frac{\mathrm{t}^{2}}{2}+\frac{\mathrm{x}^{4}}{4}+\frac{\mathrm{x}^{3} \mathrm{t}^{2}}{2}+\frac{\mathrm{x}^{2} \mathrm{t}^{3}}{2}+\frac{\mathrm{t}^{4}}{4}\right]-\mathrm{L}_{\mathrm{t}}^{-1} \frac{1}{\mathrm{~s}} \mathrm{~L}_{\mathrm{t}}\left(\mathrm{L}_{\mathrm{x}}^{-1} \frac{1}{\mathrm{~s}} \mathrm{~L}_{\mathrm{x}}\left(\mathrm{u}^{3}\right)\right)
$$

Derivation

$$
\frac{\partial^{2} \mathrm{u}}{\partial \mathrm{x} \partial \mathrm{t}} \rightarrow \frac{\partial}{\partial \mathrm{x}}\left(\frac{\partial \mathrm{u}}{\partial \mathrm{t}}\right) \rightarrow \frac{\partial \mathrm{u}}{\partial \mathrm{t}}=\mathrm{t}+\mathrm{x}^{3} \mathrm{t}+\frac{3}{2} \mathrm{x}^{2} \mathrm{t}^{2}+
$$

Differentiate equation (32) with respect to $\mathrm{x}$ :

$$
\begin{gathered}
\rightarrow \frac{\partial}{\partial \mathrm{x}}=3 \mathrm{x}^{2} \mathrm{t}+3 \mathrm{xt}^{2} \\
\therefore \frac{\partial^{2} \mathrm{u}}{\partial \mathrm{x} \partial \mathrm{t}}=3 \mathrm{x}^{2} \mathrm{t}+3 \mathrm{xt}^{2} \\
\mathrm{U}_{\mathrm{n}+1}(\mathrm{x}, \mathrm{t})=\mathrm{U}_{\mathrm{n}}(\mathrm{x}, \mathrm{t})-\int_{0}^{\mathrm{x}} \int_{0}^{\mathrm{t}} \lambda(\mathrm{t})\left(\mathrm{u}_{\mathrm{n}}\right)_{\mathrm{xt}}(\mathrm{x}, \mathrm{t})-3 \mathrm{x}^{2} \mathrm{t}-3 \mathrm{xt}^{2}+\frac{\partial^{2} \mathrm{u}}{\partial \mathrm{x} \partial \mathrm{t}}\left[\mathrm{L}_{\mathrm{t}}^{-1} \frac{1}{\mathrm{t}} \mathrm{L}_{\mathrm{t}} \mathrm{L}_{\mathrm{x}}^{-1} \frac{1}{\mathrm{t}} \mathrm{L}_{\mathrm{x}}\left(\mathrm{u}_{\mathrm{n}}\right)^{3}\right] \mathrm{dtdx} \\
\left(\mathrm{u}_{0}\right)_{\mathrm{xt}}(\mathrm{x}, \mathrm{t})=\frac{\partial}{\partial \mathrm{x}}\left(\frac{\partial \mathrm{U}_{0}}{\partial \mathrm{t}}\right) \rightarrow \frac{\partial}{\partial \mathrm{x}}(1)=0,\left(\mathrm{u}_{0}\right)_{\mathrm{xt}}(\mathrm{x}, \mathrm{t})=0 \\
\mathrm{~L}_{\mathrm{t}}^{-1} \frac{1}{\mathrm{~s}} \mathrm{~L}_{\mathrm{t}} \mathrm{L}_{\mathrm{x}}^{-1} \frac{1}{\mathrm{~s}} \mathrm{~L}_{\mathrm{x}}\left(\mathrm{u}_{0}\right)^{3}=\mathrm{L}_{\mathrm{t}}^{-1} \frac{1}{\mathrm{~s}} \mathrm{~L}_{\mathrm{t}} \mathrm{L}_{\mathrm{x}}^{-1} \frac{1}{\mathrm{~s}} \mathrm{~L}_{\mathrm{x}}(\mathrm{x}+\mathrm{t})^{3}=\frac{\mathrm{x}^{4}}{4}+\frac{\mathrm{x}^{3} \mathrm{t}^{2}}{2}+\frac{\mathrm{x}^{2} \mathrm{t}^{3}}{2}+\frac{\mathrm{t}^{4}}{4} \\
\frac{\partial}{\partial \mathrm{x}}\left(\frac{\partial \mathrm{u}}{\partial \mathrm{t}}\right)=\frac{\partial}{\partial \mathrm{x}}\left(\mathrm{x}^{3} \mathrm{t}+\frac{3}{2} \mathrm{x}^{2} \mathrm{t}^{2}+\mathrm{t}^{3}\right)=3 \mathrm{x}^{2} \mathrm{t}+3 \mathrm{xt}^{2}
\end{gathered}
$$
$\mathrm{T}$ and using Lagrange multiplier $\lambda=-1$ and then

The following equation shows the general form for the correction functional after solving the equation (36) with

Taking inverse Laplace of the equation (26):

$$
\mathrm{U}(\mathrm{x}, \mathrm{t})=\left[\mathrm{t}+\frac{\mathrm{x}^{4}}{4}+\mathrm{x}^{3} \mathrm{t}+\frac{3}{2} \mathrm{x}^{2} \mathrm{t}^{2}+\mathrm{t}^{3}\right]-\mathrm{L}_{\mathrm{x}}^{-1} \frac{1}{\mathrm{~s}} \mathrm{~L}_{\mathrm{x}}\left(\mathrm{u}^{3}\right)
$$

$$
\mathrm{u}_{\mathrm{xt}}=\frac{\partial^{2} \mathrm{u}}{\partial \mathrm{x} \partial \mathrm{t}} \rightarrow \frac{\partial}{\partial \mathrm{x}}\left(\frac{\partial \mathrm{u}}{\partial \mathrm{x}}\right) \rightarrow \text { putting } \frac{\partial \mathrm{u}}{\partial \mathrm{t}}=\mathrm{U}
$$

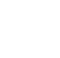




$$
\begin{gathered}
U_{1}(x, t)=U_{0}(x, t)-\int_{0}^{x} \int_{0}^{t}(0)-3 x^{2} t-3 x t^{2}+3 x^{2} t+3 x t^{2} d t d x \\
U_{1}(x, t)=x+t-0 \\
\therefore U_{1}=x+t \\
U_{1}=U_{0} \\
U_{2}=U_{1}=U_{0}=x+t \\
U_{n}=x+t \\
\therefore u(x, t)=\lim _{n \rightarrow \infty} u_{n}(x, t)=x+t
\end{gathered}
$$

The solution of example 1 showed that the exact solution of this equation is achieved after just one iteration since lim $\mathrm{u}_{\mathrm{n}}=\mathrm{u}_{0}=\mathrm{x}+\mathrm{t}$. The same exact solution was achieved using other solution methods used by other authors [1, 6-10, 14], yet, these methods required more than four iterations while the proposed LS-VIM method achieved the exact solution after a single iteration only. This in fact has a great impact in decreasing the effort of processing iteration in complex problems.

\subsection{Example 2}

In this example, a more complex second order Goursat equation with two variables is solved to evaluate the proposed method. Consider the following nonlinear inhomogeneous Goursat Problem

$$
\begin{gathered}
u_{x t}=-u^{2}+e^{2 x}+e^{2 t}+2 \\
u(x, 0)=1+e^{x}, u(0, t)=1+e^{t}, u(0,0)=2
\end{gathered}
$$

Solution:

$$
u_{x t}=\frac{\partial^{2} \mathrm{u}}{\partial \mathrm{x} \cdot \partial \mathrm{t}} \rightarrow \frac{\partial}{\partial \mathrm{x}}\left(\frac{\partial}{\partial \mathrm{t}}\right)
$$

Putting $\frac{\partial \mathrm{u}}{\partial \mathrm{t}}=\mathrm{U}$

$$
\frac{\partial U}{\partial x}=-u^{2}+e^{2 x}+e^{2 t}+2 e^{x+t}
$$

Taking the Laplace transform with respect to $\mathrm{x}$ :

$$
\begin{gathered}
\mathrm{L}_{\mathrm{x}}\left(\frac{\partial \mathrm{U}}{\partial \mathrm{x}}\right)=\mathrm{L}_{\mathrm{x}}\left[\mathrm{e}^{2 \mathrm{x}}+\mathrm{e}^{2 \mathrm{t}}+2 \mathrm{e}^{\mathrm{x}+\mathrm{t}}\right]-\mathrm{L}_{\mathrm{x}}\left[\mathrm{u}^{2}\right] \\
\mathrm{Su}(\mathrm{s}, \mathrm{t})-\mathrm{U}(\mathrm{o}, \mathrm{t})=\mathrm{L}_{\mathrm{x}}\left[\mathrm{e}^{2 \mathrm{x}}+\mathrm{e}^{2 \mathrm{t}}+2 \mathrm{e}^{\mathrm{x}+\mathrm{t}}\right]-\mathrm{L}_{\mathrm{x}}\left[\mathrm{u}^{2}\right] \\
\mathrm{Su}(\mathrm{s}, \mathrm{t})-\left(1+\mathrm{e}^{\mathrm{t}}\right)=\left[\frac{1}{\mathrm{~s}-2}+\mathrm{e}^{2 \mathrm{t}}+\frac{2 \mathrm{e}^{\mathrm{t}}}{\mathrm{s}-1}\right]-\mathrm{L}_{\mathrm{x}}\left(\mathrm{u}^{2}\right)
\end{gathered}
$$

Divide equation (48) by S:

$$
\mathrm{U}(\mathrm{s}, \mathrm{t})=\frac{1+\mathrm{e}^{\mathrm{t}}}{\mathrm{s}}+\frac{1}{\mathrm{~s}(\mathrm{~s}-2)}+\frac{\mathrm{e}^{2 \mathrm{t}}}{\mathrm{s}}+\frac{2 \mathrm{e}^{\mathrm{t}}}{\mathrm{s}(\mathrm{s}-1)}-\frac{1}{\mathrm{~s}} \mathrm{~L}_{\mathrm{x}}\left(\mathrm{u}^{2}\right)
$$

Applying inverse Laplace transform for equation (49):

$$
\begin{aligned}
& U(x, t)=1+e^{t}+e^{2 x}+e^{2 t}+2 e^{x+t}-L_{x}^{-1} \frac{1}{s} L_{x}\left(u^{2}\right) \\
& \frac{\partial U(x, t)}{\partial t}=1+e^{t}+e^{2 x}+e^{2 t}+2 e^{x+t}-L_{x}^{-1} \frac{1}{S} L_{x}\left(u^{2}\right)
\end{aligned}
$$

Taking Laplace transform with regards to t:

$$
\begin{aligned}
& S U(x, t)-U(x, 0)=L_{t}\left[1+e^{t}+e^{2 x}+e^{2 t}+2 e^{x+t}\right]-L_{t} L_{x}^{-1} \frac{1}{S} L_{x}\left(u^{2}\right. \\
& S U(x, t)-\left(1+e^{x}\right)=L_{t}\left[1+e^{t}+e^{2 x}+e^{2 t}+2 e^{x+t}\right]-L_{t} L_{x}^{-1} \frac{1}{S} L_{x}\left(u^{2}\right) \\
& S U(x, t)=\left(1+e^{x}\right)+\left[1+\frac{1}{s-1}+e^{2 x}+\frac{1}{s-2}+\frac{2 e^{x}}{S-1}\right]-\left[L_{t} L_{x}^{-1} \frac{1}{S} L_{x}\left(u^{2}\right)\right]
\end{aligned}
$$

Divide by S:

$$
\mathrm{U}(\mathrm{x}, \mathrm{t})=\frac{\left(1+\mathrm{e}^{\mathrm{x}}\right)}{\mathrm{S}}+\left[\frac{1}{\mathrm{~S}}+\frac{1}{\mathrm{~S}(\mathrm{~S}-1)}+\frac{\mathrm{e}^{2 \mathrm{x}}}{\mathrm{S}}+\frac{1}{\mathrm{~S}(\mathrm{~S}-2)}+\frac{2 \mathrm{e}^{\mathrm{x}}}{\mathrm{S}(\mathrm{S}-1)}\right]-\left[\frac{1}{\mathrm{~S}} \mathrm{~L}_{\mathrm{t}} \mathrm{L}_{\mathrm{x}}^{-1} \frac{1}{\mathrm{~S}} \mathrm{~L}_{\mathrm{x}}\left(\mathrm{u}^{2}\right)\right]
$$

Taking Inverse Laplace transform:

$$
\begin{gathered}
\mathrm{U}(\mathrm{x}, \mathrm{t})=1+\mathrm{e}^{\mathrm{x}}+1+\mathrm{e}^{\mathrm{t}}+\mathrm{e}^{2 \mathrm{x}}+\mathrm{e}^{2 \mathrm{t}}+2 \mathrm{e}^{\mathrm{x}+\mathrm{t}}-\mathrm{L}_{\mathrm{t}}^{-1}\left[\frac{1}{\mathrm{~s}} \mathrm{~L}_{\mathrm{t}} \mathrm{L}_{\mathrm{x}}^{-1} \frac{1}{\mathrm{~s}} \mathrm{~L}_{\mathrm{x}}\left(\mathrm{u}^{2}\right)\right] \\
\therefore \frac{\partial^{2} \mathrm{U}(\mathrm{x}, \mathrm{t})}{\partial \mathrm{x} \partial \mathrm{t}} \rightarrow \frac{\partial \mathrm{U}}{\partial \mathrm{t}}=\mathrm{e}^{\mathrm{t}}+2 \mathrm{e}^{2 \mathrm{t}}+2 \mathrm{e}^{\mathrm{t}+\mathrm{x}}-\mathrm{L}_{\mathrm{t}}^{-1}\left[\frac{1}{\mathrm{~s}} \mathrm{~L}_{\mathrm{t}} \mathrm{L}_{\mathrm{x}}^{-1} \frac{1}{\mathrm{~s}} \mathrm{~L}_{\mathrm{x}}\left(\mathrm{u}^{2}\right)\right] \\
\frac{\partial \mathrm{U}}{\partial \mathrm{x}}=2 \mathrm{e}^{\mathrm{x}+\mathrm{t}}-\frac{\partial^{2} \mathrm{u}}{\partial \mathrm{x} \partial \mathrm{t}}\left[\mathrm{L}_{\mathrm{t}}^{-1} \frac{1}{\mathrm{~s}} \mathrm{~L}_{\mathrm{t}} \mathrm{L}_{\mathrm{x}}^{-1} \frac{1}{\mathrm{~s}} \mathrm{~L}_{\mathrm{x}}\left(\mathrm{u}^{2}\right)\right] \\
\left.\mathrm{U}_{\mathrm{xt}}=2 \mathrm{e}^{\mathrm{x}+\mathrm{t}}-\frac{\partial^{2} \mathrm{u}}{\partial \mathrm{x} \partial \mathrm{t}}\left[\mathrm{L}_{\mathrm{t}}^{-1} \frac{1}{\mathrm{~s}} \mathrm{~L}_{\mathrm{t}} \mathrm{L}_{\mathrm{x}}^{-1} \frac{1}{\mathrm{~s}} \mathrm{~L}_{\mathrm{x}}\left(\mathrm{u}^{2}\right)\right)\right]
\end{gathered}
$$


By substituting (59) in the correction function and using the Lagrange multiplier $\lambda=-1$, also continuing by selecting $\mathrm{U}_{0}=\mathrm{e}^{\mathrm{t}}+\mathrm{e}^{\mathrm{x}}$ from the used boundary conditions we get:

$$
\begin{gathered}
\left.U_{n+1}(x, t)=U_{0}(x, t)-\int_{0}^{x} \int_{0}^{t}\left(\left(u_{n}\right)_{x t}(x, t)-2 e^{x+t}-\frac{\partial^{2} u}{\partial x \partial t}\left[L_{t}^{-1} \frac{1}{s} L_{t} L_{x}^{-1} \frac{1}{s} L_{x}\left(u^{2}\right)\right)\right]\right) d s d x \\
U_{1}=e^{t}+e^{x}- \\
\therefore U_{1}=e^{t}+e^{x} \\
U_{1}=U_{0} \\
U_{2}=U_{1} \\
U_{n}=e^{t}+e^{x} \\
\therefore u(x, t)=\lim _{n \rightarrow \infty} u_{n}(x, t) \\
u(x, t)=e^{t}+e^{x}
\end{gathered}
$$

Similar to the case presented in example 1, the exact solution of the equation is obtained after a single iteration when LS-VIM is use. The solution is similar to the results obtained by other authors $[1,6-10,14]$ except the fact that in the mentioned references above the exact solution was achieved after many iterations. Despite the difficulty of achieving the exact solutions of such equations compared to the equation in Example 1, the exact solution of this equation was achieved after only one iteration using the proposed method.

\section{Conclusion}

In this paper, the Laplace substitution-Variational iteration method is introduced and used to solve Goursat problem. The results obtained from solving the two examples showed that the proposed method can provide the exact solution by only a single iteration. Comparing results with other methods in solving the same equation, can be concluded that this method can minimize the number of iteration rapidly to reach the exact solution. The proposed method is considered as a strong and reliable mathematical tool as compared to other methods to solve more complicated/advanced nonlinear partial differential equations.

\section{References}

[1] Ahmad J, Mushtaq M. Exact Solution of Linear and Non-linear Goursat Problems. Univers J Comput Math. 2015; 3 (1): 14-17..

[2] A. AL-Fayadh and H. Khawwan, "Variation Iteration Transform Method For Solving Burger and Coupled Burgers eqution," ARPN J. Eng. Appl. Sci., vol. 12, no. 23, pp. 6926-6932, 2017.

[3] Evans D., Sanusi B. Numerical Solution of the Goursat Problem By A Nonlinear Trapezoidal Forula. Appl Math Lett. 1988; I (3): 221-223.

[4] Handibag S., Karande BD. An Application for Nonlinear Partial Differential Equations Involving Mixed Partial Derivatives by Laplace Substitution Method. In: 10th International Conference on Mathematical Problems in Engineering, Aerospace and Science. AIP Conf. Proc. Vol 1637; 384-394, (2014).

[5] J.T. Day, A Runge-Kutta method for the numerical solution of the Goursat problem in hyperbolic partial differential equations.

[6] Muhammad Aslam Noor ,Syed Tauseef Mohyud-Din.Modified Variational Iteration Method for Goursat and Laplace Problems.Appl Sciences Journal 4 (4): 487-498, 2008.

[7] SharafMohmoud, Mohamed Gubara. Reduced Differential Transform Method.Appl Math, 2016, 7, 1049-1056

[8] Taghvafard H, Erjaee GH. Two-dimensional Differential Transform Method for Solving Linear and Non-linear Goursat Problem. Int J Math Comput Phys Electr Comput Eng. 2010; 4 (3):432-435.

[9] Usman M, Zubair T, Ali U, Mohyud-din ST. On Goursat Problems. Int J Mod Math Sci. 2012; 3 (3): 63-76.

[10] Wazwaz A. The variational iteration method for a reliable treatment of the linear and the nonlinear Goursat problem. Appl Math Comput. 2007; 193: 455-462

[11] Handibag S, Karande BD. Laplace Substitution Method for Solving Partial Differential Equations Involving Mixed Partial Derivatives. Int J Comput Eng Res. 2012; 2 (4): 1049-1052.

[12] Handibag S, Karande B. LAPLACE SUBSTITUTION METHOD FOR SOLVING PARTIAL DIFFERENTIAL EQUATIONS INVOLVING MIXED PARTIAL DERIVATIVES. Int J Pure Appl Math. 2012; 78 (7): 973-979.

[13] Handibag S., Karande B. Laplace Substitution Method for $\mathrm{n}$ th Order Linear and Non-Linear PDE' s Involving Mixed Partial Derivatives. Int Res J Eng Technol. 2015; 2 (9): 378-388.

[14] Pandey PK. A Finite Difference Method for Numerical Solution of Goursat Problem of Partial Differential Equation. Open Access Libr J. 2014; 1: 1-6.

[15] Ramadan MA, Raslan KR, Hadhoud AR, Mesrega AK. A Substitution Method for Partial Differential Equations Using Ramadan Group Integral Transform. Asian Res J Math. 2017; 7 (4): $1-10$. 